\title{
Detection of Cancer in Intestine using CT Images
}

\author{
Sushmitha $\mathbf{S}^{1}$, Shilpa Karemuragi ${ }^{2}$, Syed Sumeer ${ }^{3}$, Shiva Kumar $C^{4}$ \\ Student, ECE Department, GMIT, Mandya, India ${ }^{1,2,3}$ \\ Asst Prof, ECE Department, GMIT, Mandya, India ${ }^{4}$
}

\begin{abstract}
Colorectal cancer is the most commonly diagnosed cancer leading to death in men and women. Computed tomography (CT) imaging technique established itself as the primary method for detection and staging in patients with colorectal cancer. In this work CT image of colorectal cancer are used to detect the area and staging the cancer. This work describes simple algorithm for pre-processing, clustering \& post processing of CT images. This work uses image enhancement techniques and clustering using Fuzzy C-means algorithm which is implemented on MATLAB.
\end{abstract}

Keywords: Colorectal cancer, CLAHE, Fuzzy C-Mean, Computed tomography (CT), Computed tomography colonography (CTC).

\section{INTRODUCTION}

Intestinal cancer is cancer that starts in the colon or the rectum inside the large intestine. These cancers can also be referred to separately as colon cancer or rectal cancer, depending on where they start. It is the second leading cause of death among cancers.

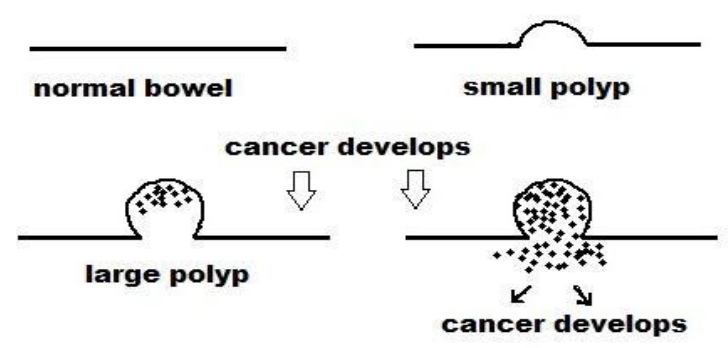

Fig 1: Levels of cancer development

Given the fact that intestinal cancer is a largely preventable disease through routine detection, removal of polyps and intestinal cancer prevention has now moved to the forefront. Levels of intestine cancer development from the normal bowel are shown in Figure 1. Most intestinal cancers begin as polyps. As polyps enlarge, they are more likely to develop into a cancer as shown in Figure 2, which has the ability to disseminate through the body. The larger the polyp, the greater the probability that the polyp will have undergone malignant change and contain cancer.

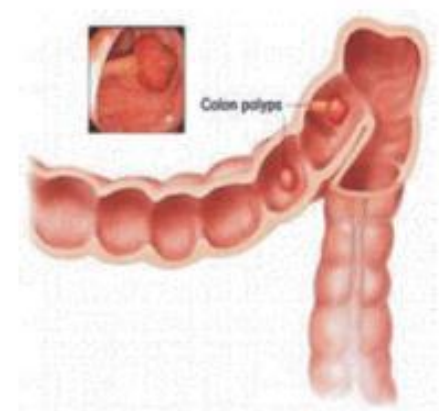

Fig 2: The polyps on the colon wall.

\section{DESIGN METHODOLOGY}

The below figure shows the complete block diagram of the processed work. CT image in the form of JPEG will become the input for the proposed work. 


\section{International Journal of Innovative Research in} Electrical, Electronics, Instrumentation and Control Engineering

\section{ISO 3297:2007 Certified}

Vol. 5, Issue 6, June 2017

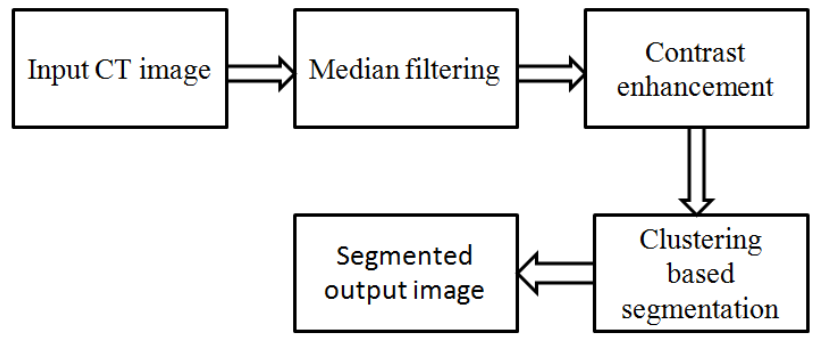

Fig. 3: Block diagram of processed work

The median filter is a nonlinear digital filtering technique, often used to remove noise. Such noise reduction is a typical preprocessing step to improve the results of later processing (for example, edge detection on an image).

Image enhancement techniques have been widely used in many application of image processing where the subjective quality of image is important for human interpretation. Contrast is an important factor in any subjective evaluation of image quality. Adaptive histogram equalization (AHE) is a computer image processing technique used to improve contrast in images. It differs from ordinary histogram equalization in the respect that the adaptive method computes several histograms, each corresponding to a distinct section of the image, and uses them to redistribute the lightness value of the image. It is therefore suitable for improving the local contrast and enhancing the definitions of edges in each region of CT image. AHE has a tendency to over amplify noise in relatively homogeneous regions of an image. A variant of adaptive histogram equalization called contrast limited adaptive histogram equalization (CLAHE) prevents this by limiting the amplification.

The purpose of Image Segmentation is to cluster pixels into salient image regions. We present Fuzzy C-Means (FCM) cluster segmentation algorithm based on modified membership that incorporates spatial information into the membership function for clustering. The spatial function is the weighted summation of the membership function in the neighborhood of each pixel under consideration.

\section{RESULTS AND IMPLEMENTATION}

The results have been simulated in MATLAB. The images have been taken from a private diagnostic centre and the segmentation is done by fuzzy c means which classifies the image into clusters. It is used to evaluate the efficiency of the clusters based on each iteration and the computational time required to simulate the image.

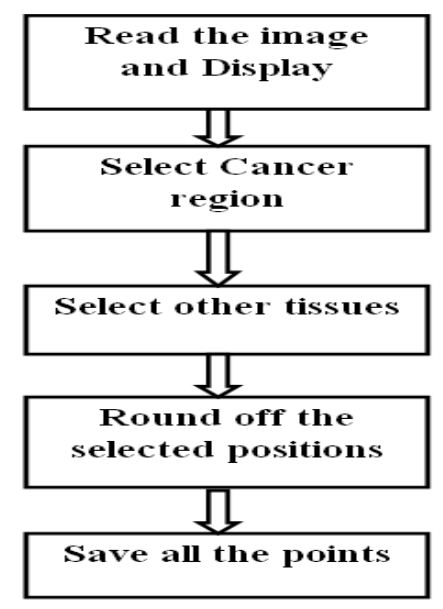

Fig 4: Flow diagram of stage 1

Stage 1: The first stage shown in figure 4. The algorithm requires the input CT images in JPG format. If the CT images collected from radiologist are in DICOM format, they must be converted into JPG format. The CT image of colorectal cancer in JPG format is read and displayed. The cancer regions are selected by marking points using left click of the mouse. After done with selection of sufficient number of points, it is terminated by a right click. To proceed further with selection of other tissue regions, the same above procedure is repeated. Once all the selection of points for the entire region is done, the selected positions are rounded off to nearest integer values. Every point is saved after rounding off. 


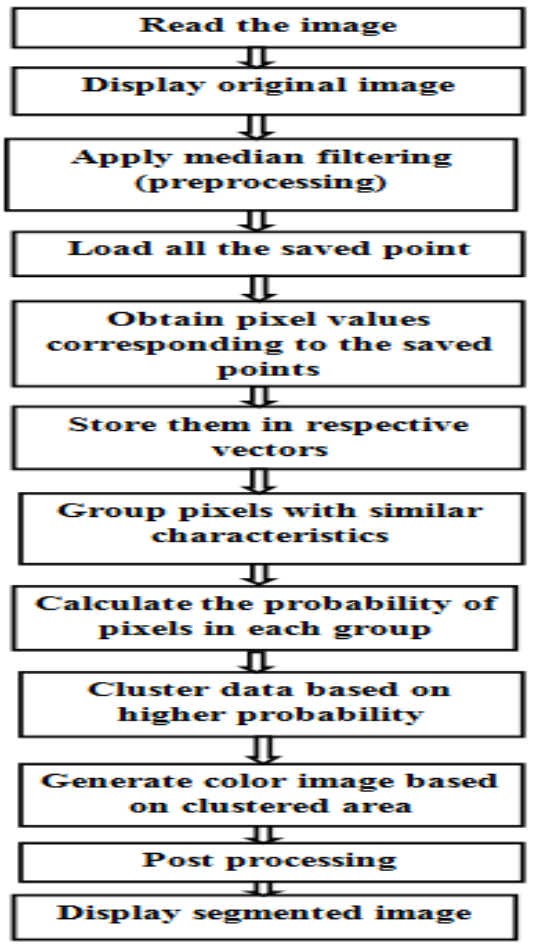

Fig 5: Flow diagram of stage 2

Stage 2: The second stage is all about separating the selected cancer points from the surrounding healthy tissues. The original image is read and displayed as shown in figure 5. it is subjected to $3 \times 3$ median filtering for pre-processing of the image. In order to implement clustering algorithm, all the saved points during the first stage are loaded. The pixel values corresponding to the saved points are extracted and are in respective vectors. Those pixels exhibiting similar characteristics are grouped together. A pixel can be considered in more than one group. If it is present in multiple groups, the probability of pixel in each group is computed. The data is clustered based on highest probability. A colour image is generated based on clustered area. It is followed by post processing techniques using morphological operations like dilation and erosion followed by holes-filling. Post processing is carried out only if blurring of images is noticed in the output of segmentation. The segmentation output images considered during the implementation of proposed work did not show any blurring on the edges and provided satisfactory results. Hence post processing was not carried out as it will not create much difference in the results if no blurring is present the final segmented image is displayed on the monitor screen. The figure 6 shows the output of the stage 1 . Here the green colour selection for cancer tissue and other colour is given to healthy tissues.

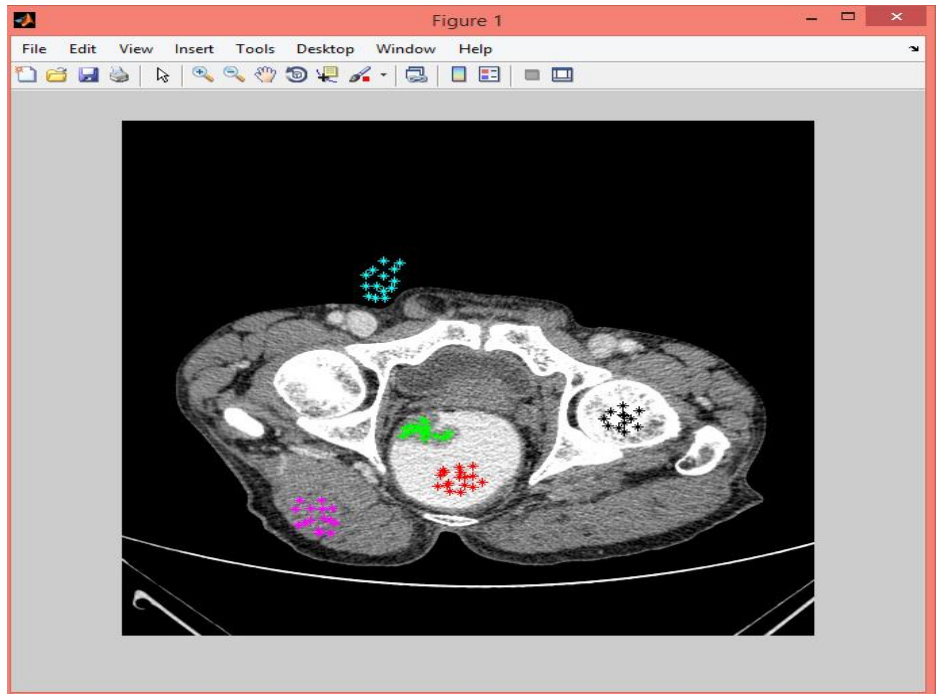

Fig 6: Stage 1 output 
IJIREEICE

\section{International Journal of Innovative Research in} Electrical, Electronics, Instrumentation and Control Engineering

ISO 3297:2007 Certified

Vol. 5, Issue 6, June 2017

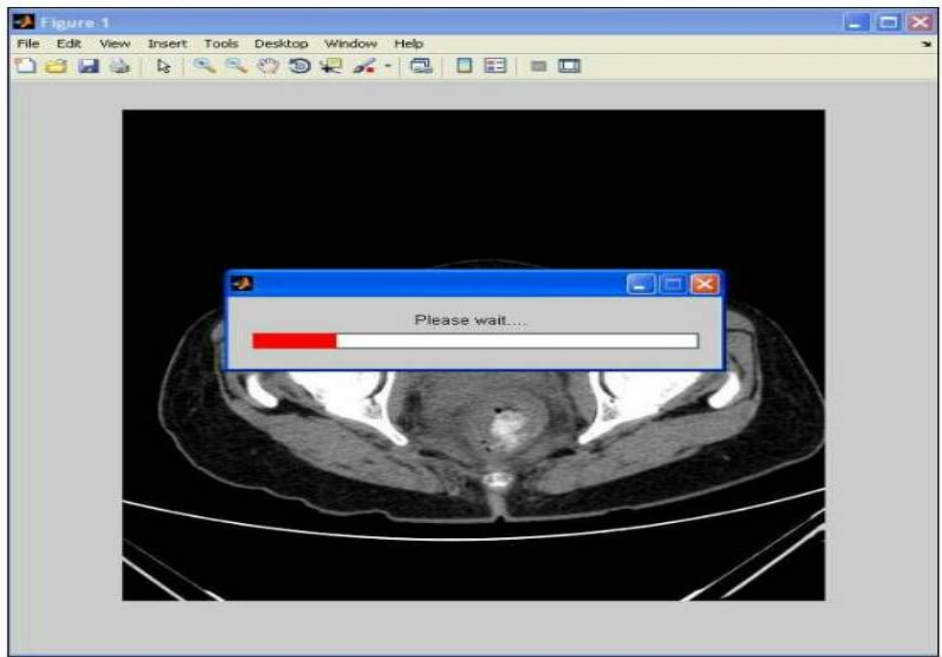

Fig 7: Execution status

The input for the stage 2 will be same as the input image used in stage 1 . Soon after execution of stage 2 begins, a window appears along with the input image that displays the status of execution carried out at every instant of time, which is shown in figure 7.
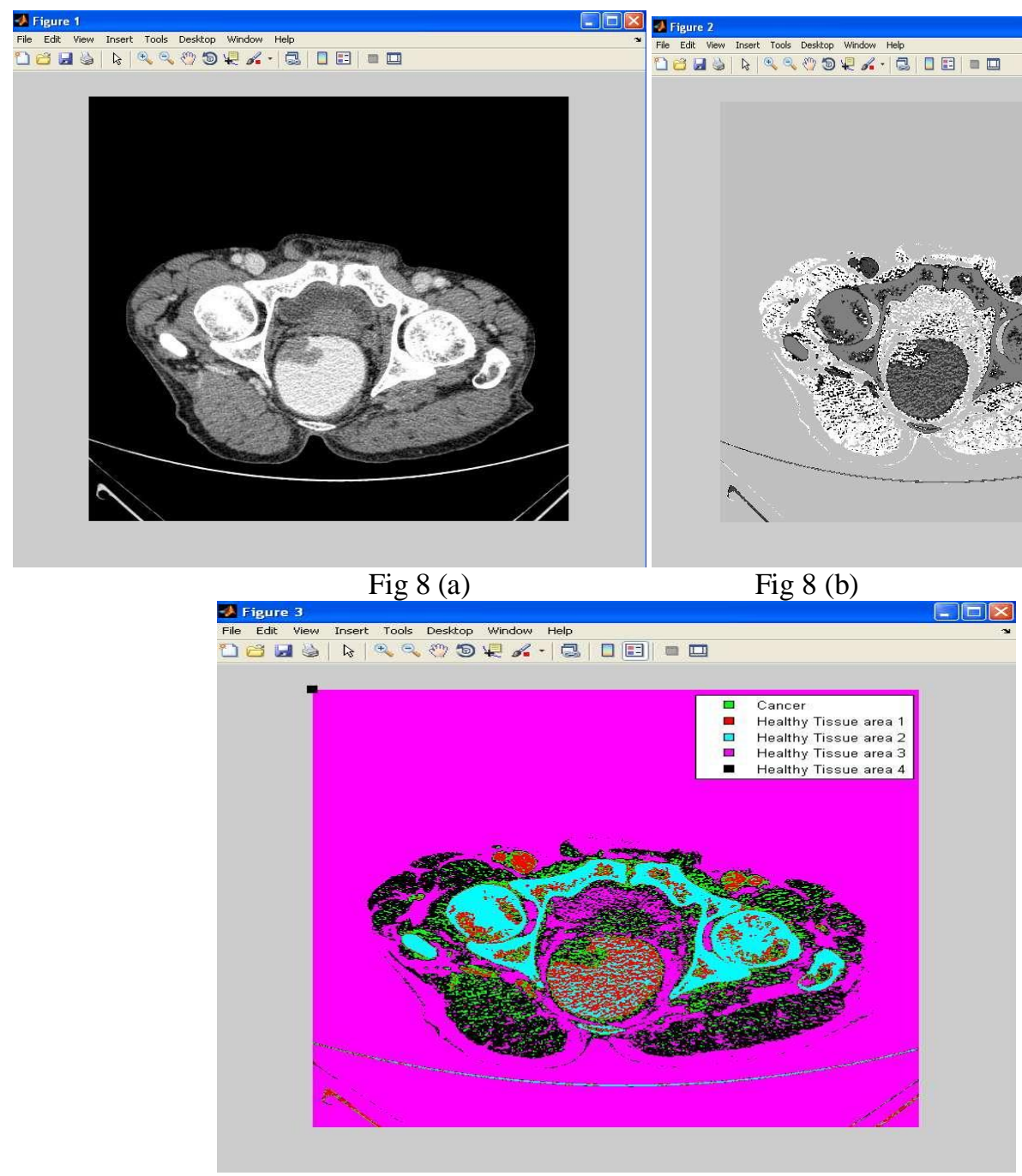

Fig 8 (c)

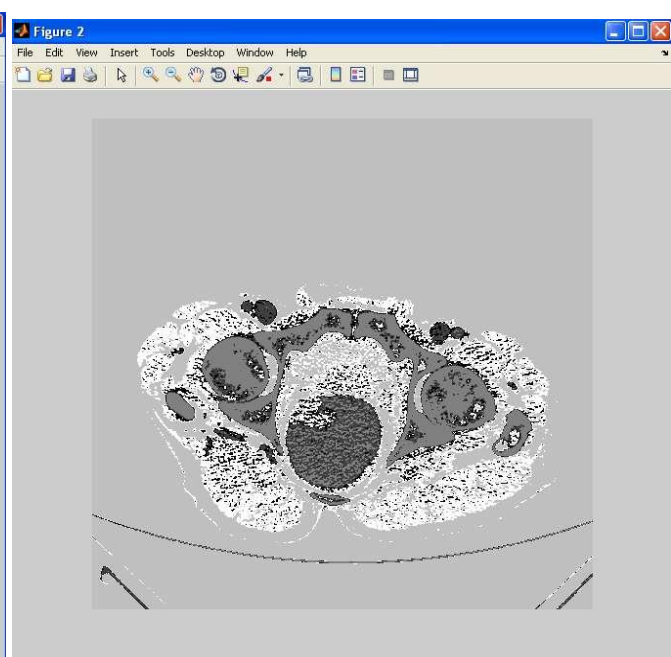

Fig 8 (b) 
The figure 8 shows the colon cancer detection at the initial stage. The figure 8(a) shows the median filtered CT image given as input. The figure 8(b) shows the segmented output that in the gray scale format. The figure 8(c) shows the coloring output of the segmented image, here the green color indicates the cancer region. The less green color indicates cancer at in the initial stage, in this stage cancer can be curable.

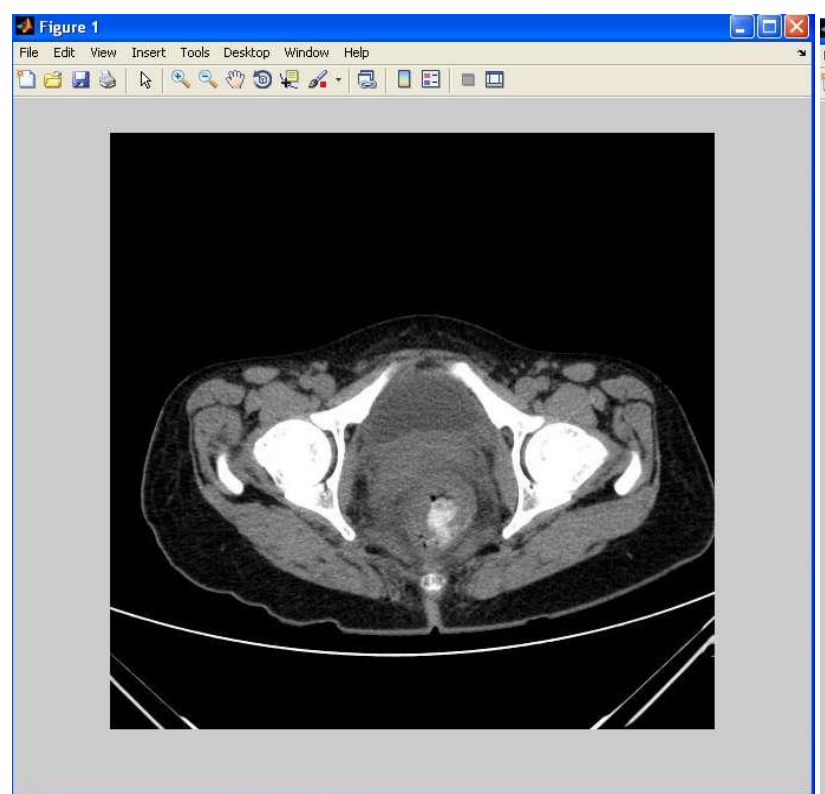

Fig 9(a)

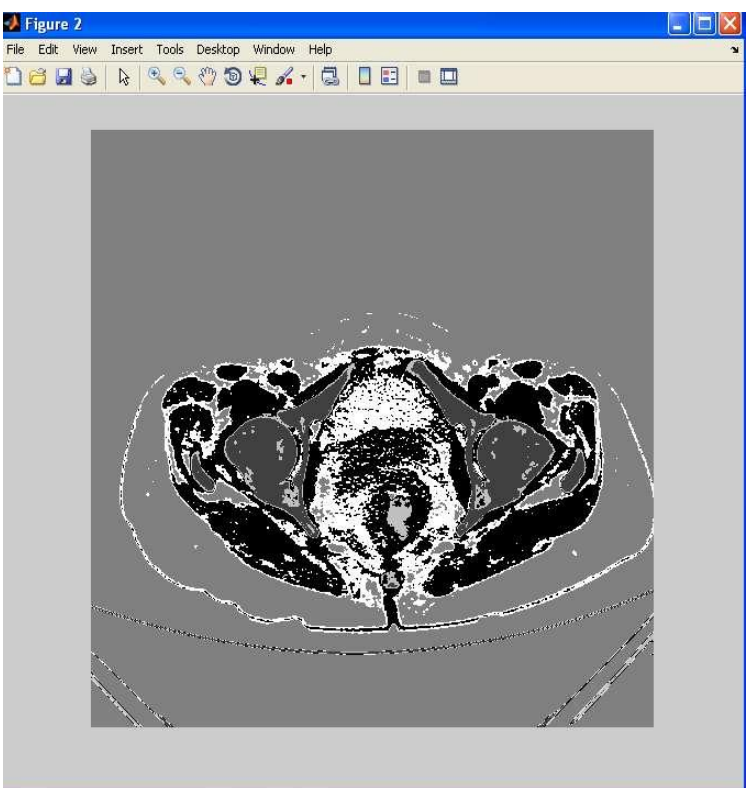

Fig 9(b)

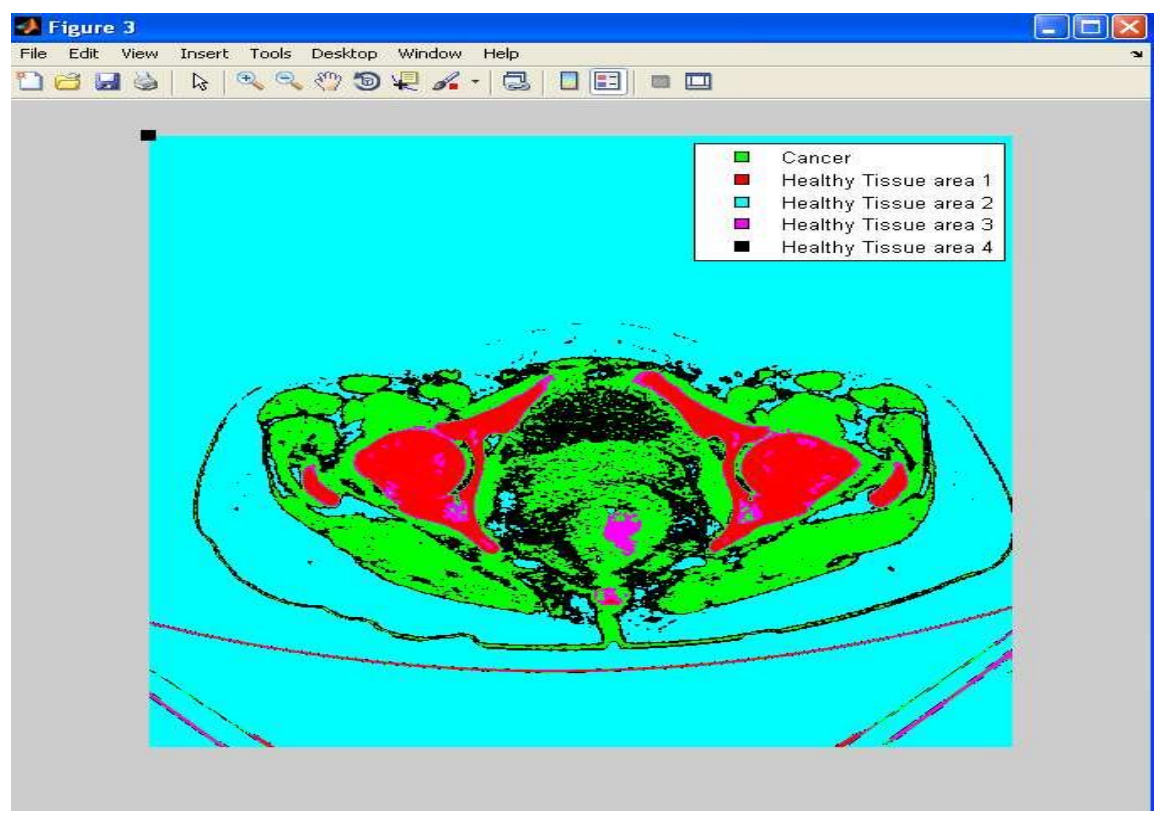

Fig 9(c)

The figure 9 shows the colon cancer detection at the final stage. The figure 9(a) shows the median filtered CT image given as input. The figure 9(b) shows the segmented output that in the gray scale format. The figure 9(c) shows the coloring output of the segmented image, here the green color indicates the cancer region. The more green color indicates cancer at in the final stage, in this stage cancer cannot be curable.

\section{CONCLUSION}

The proposed work has an intention to provide a good solution towards using the present technology in identification of intestinal cancer in the beginning stages so as to put the patient out of danger zone. 


\section{IJIREEICE

An attempt is made to bridge the gap between the radiologist and cancer specialist so that the patient gets the best treatment.

\section{REFERENCES}

[1] Xiaoyun Yang, Xujiong Ye, Member, IEEE and Greg Slabaugh, Senior Member, IEEE."Multi-Label Region Classification and Semantic Linking for Colon Segmentation in CT Colonography".

[2] G. Shantha Lakshmi,MPhil, Prof. B. Mohamed FaizeBasha, MCA., M.Phil. (2014). "Colorectal Cancer Detection using Image Processing based on IGVF Mode"l. e-ISSN: 2278-0661, p- ISSN: 2278-8727Volume 16, Issue 2, Ver. III (Mar-Apr. 2014 ), PP $39-43$.

[3] Giacomo Puppa, Graeme Poston, Per Jess, Guy F Nash, Kenneth Coenegrachts(2013). Axel Stang Staging colorectal cancer with the TNM 7th: "The presumption of innocence when applying the M category". World J Gastroenterol 2013 February 28; 19(8): 1152-1157 ISSN 1007-9327 (print) ISSN 2219-2840.

[4] Karen M. Horton, MD • Ross A. Abrams, MD • Elliot K. Fishman, MD.(2000). "Spiral CT of Colon Cancer: Imaging Features and Role in Management". RadioGraphics 2000; 20:419-430.

[5] Hong-xia Gong, Ke-bei Zhang, Lian-Ming Wu, Brian F. Baigorri, Yan Yin, Xiaochuan Geng, Jian-Rong Xu, Jiong Zhu.(2016). "Dual Energy Spectral CT Imaging for Colorectal Cancer Grading: A Preliminary Study”. PLOS ONE DOI:10.1371/journal.pone.0147756 February 9, 2016.

[6] Valentin Ignatov, Nikola Kolev, Anton Tonev, Shteryu Shterev, Elitsa Encheva, Tanya Kirilova, Tsvetelina Teneva and Krassimir Ivanov. "Diagnostic Modalities in Colorectal Cancer -Endoscopy, Ct and Pet Scanning, Magnetic Resonance Imaging (Mri),Endoluminal Ultrasound and Intraoperative Ultrasound". dx.doi.org/10.5772/57508.

[7] I-Ha Lao Yen-Jen Wang Chee-Wai Mak1 Wen-Sheng Tzeng Reng-Hong Wu. "Accuracy of Computed Tomography in Staging the Tumor Invasion Status of Colon Cance"r. J Radiol Sci 2012; 37: 51-57.

[8] Yan Xu, Jun-Yan Zhu, Eric Chang and Zhuowen Tu.” Multiple Clustered Instance Learning for Histopathology Cancer Image Classification, Segmentation and Clustering".

[9] Dongqing Chen, Hossam Abd El Munim and Aly A. Farag. "Segmentation of Colon in Tagged CT Colonography Using Adaptive Level Sets Method". 